\title{
Pregnancy and Kidney transplantation
}

\section{A R T I C L E I N F O}

\section{Article Type}

Analytical Review

\section{Authors}

Robatmoradi N.* $M D$
How to cite this article Robatmoradi N. Pregnancy and Kidney transplantation. Sarem Journal of Reproductive Medicine. 2017;1(2):69-72.
*"Hasheminehhad Hospital" and "Sarem Fertility \& Infertility Research Center (SAFIR)", Tehran, Iran

\section{Correspondence}

Address: Sarem Women's Hospital, Basij Square, Phase 3, Ekbatan Town, Tehran, Iran. Postal Code: 1396956111

Phone: +98 (21) 44670888

Fax: +98 (21) 44670432

nr_moradi@yahoo.com

\section{Article History}

Received: January 25, 2016

Accepted:April20,2016

ePublished: June 15, 2017

\section{A B S T R A C T}

Introduction The first woman, who received kidney transplantation from her identical twin sister, gave birth to a healthy baby on the tenth of March 1958 without immunosuppressive consumption. This led to an important point to prove, and it was that, after kidney transplantation, fertility is also possible, and normal kidney function alone is sufficient to withstand pregnancy and the proximity of the uterus to the kidneys will not cause mechanical problems. Obviously, women who are undergoing dialysis are experiencing significant decrease fertility, and have been shown that kidney transplantation will be able fertility in these women. In this study, the important sources and Guideline about kidney transplantation and pregnancy were studied, and according to researcher's experiences, the contents were summarized and presented.Conclusion An important issue that women kidney transplant should know, it is that pregnancy should be postponed at least one year and preferably 2 years after transplantation. There is the possibility of different complications for this category of women and pregnancy complications after the transplant should be considered. Therefore, it is recommended that a proper periodic evaluation be made during pregnancy, in a special way, under the supervision of a urologist and a perinatologist.

Keywords Kidney Transplantation; Pregnancy; Women; Fertility 
حاملهشدن يس إز يِيوند كليه يك قانون كلى نيست. در يك ريك

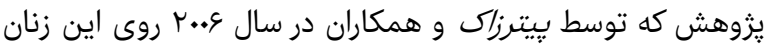

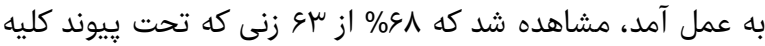

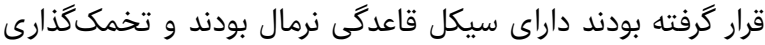

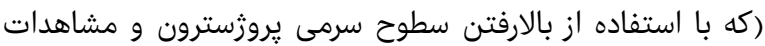

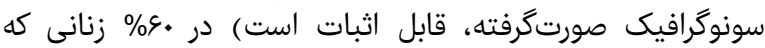

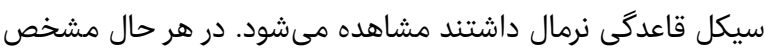

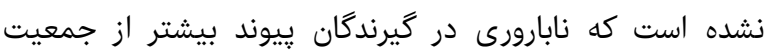

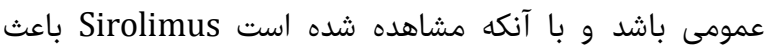

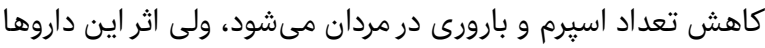

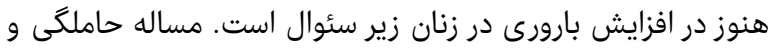

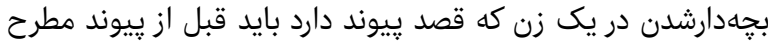

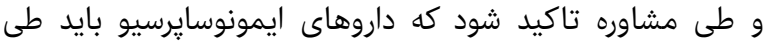

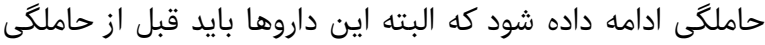

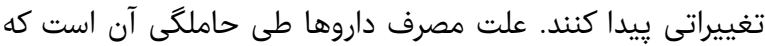

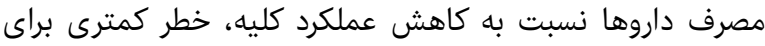

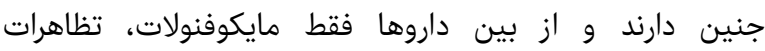

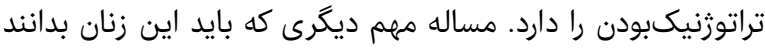

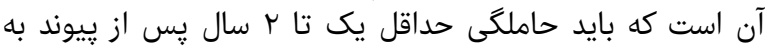

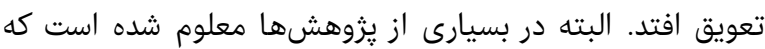

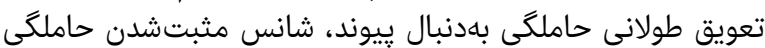

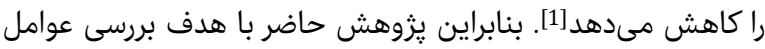

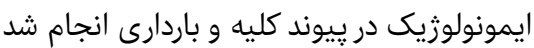

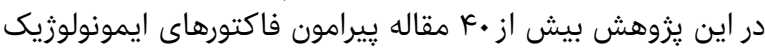

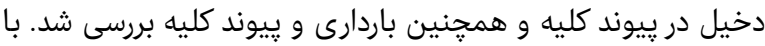

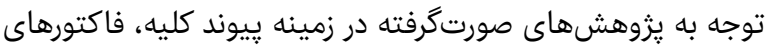

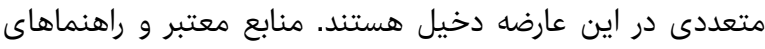

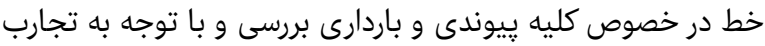

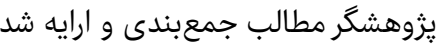

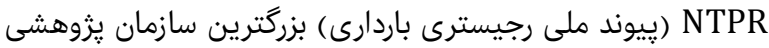

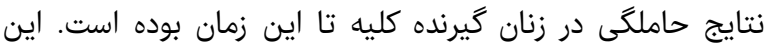

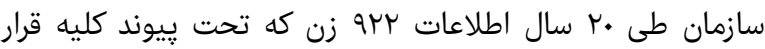

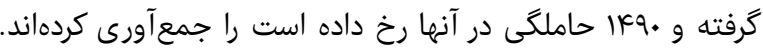

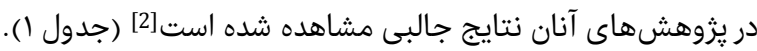

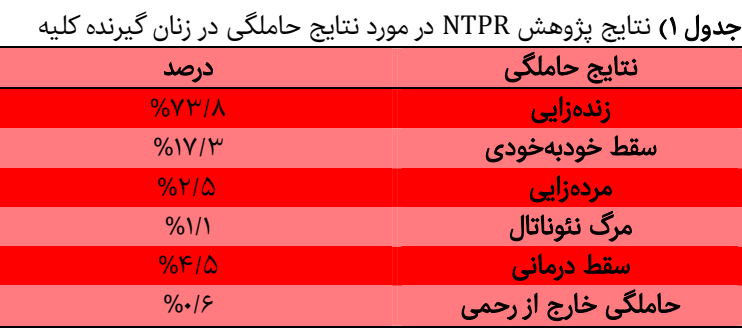

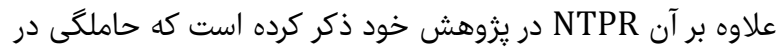

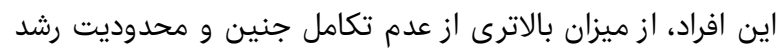

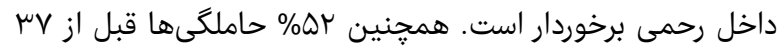
هفته خاتمه بيدا كرده است.

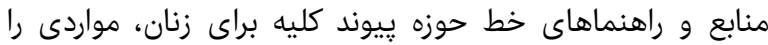

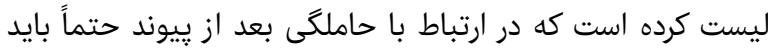

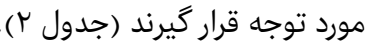

\section{باردارى و ي ييوند كليه}

MD "نادر رباطمرادى

"بيمارستان هاشمىنزاداد" و "مركز تحقيقات بارورى و نابارورى صارم"، بيمارستان فوق تخصصى صارم، تهران، ايران

קقذيده

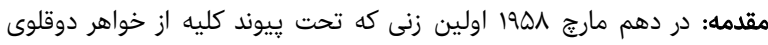

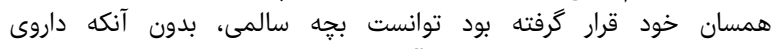

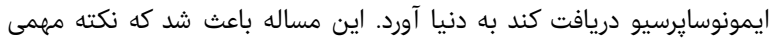

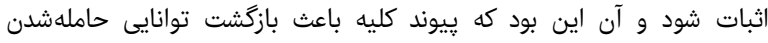

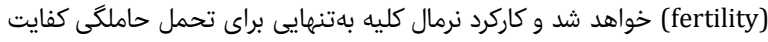

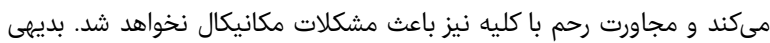

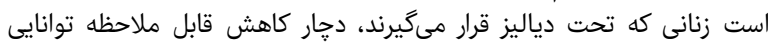

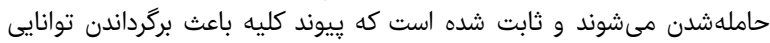

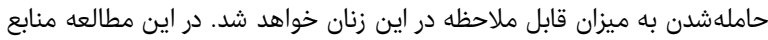

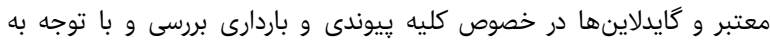

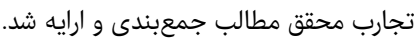

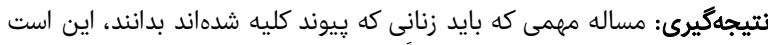

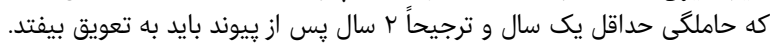

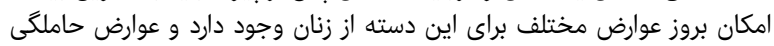

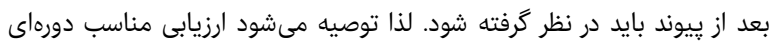

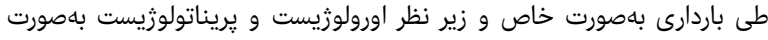

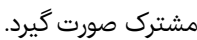
كليدوازمها: بيوند كليه، باردارى، زنان، توانايى حامله شدن

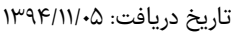

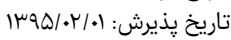

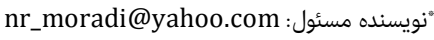

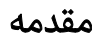

در دهم مارج سال 1901 اولين زنى كه تحت بِ بيوند كليه از خواهر

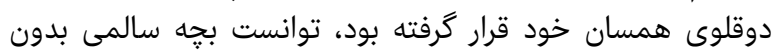

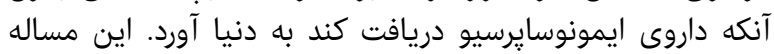

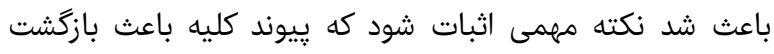

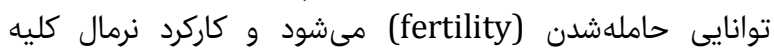

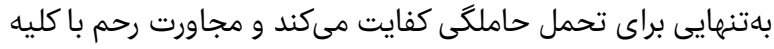

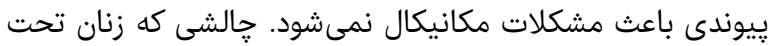

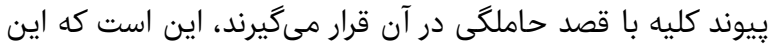

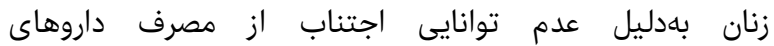

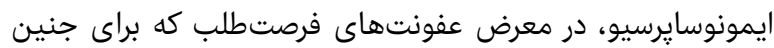

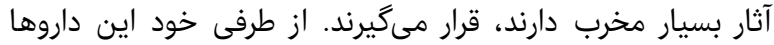

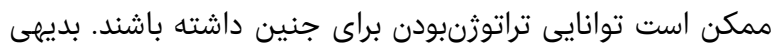

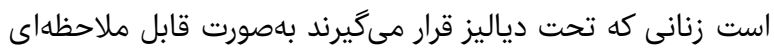

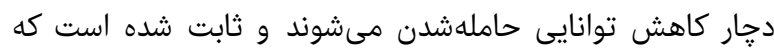

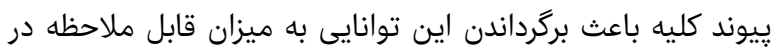

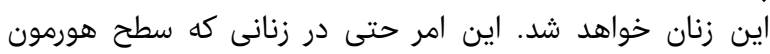

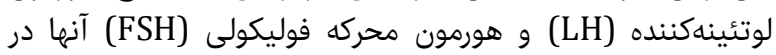

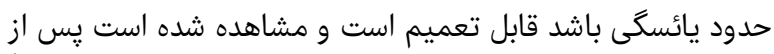

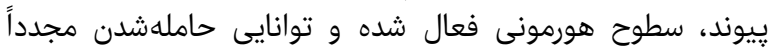

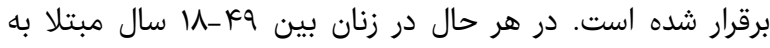
(End-Stage Renal Disease) ESRD

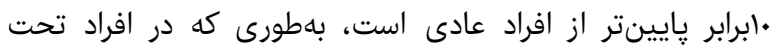

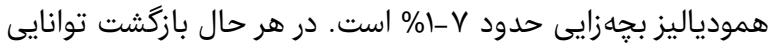


آنزيم تبديلكننده آنزيوتانسين) و بلوكرهاى گيرنده آنزيوتانسينها و آتنولول استفاده كرد.

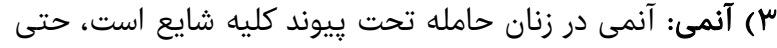

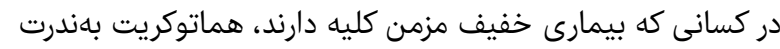

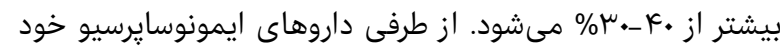

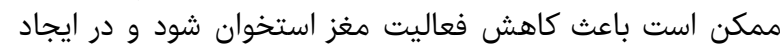

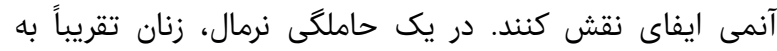

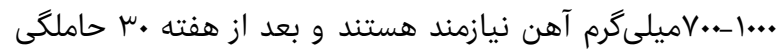

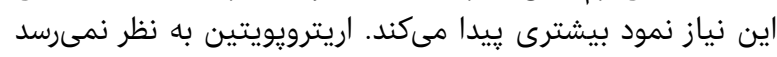

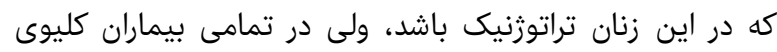

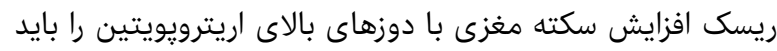

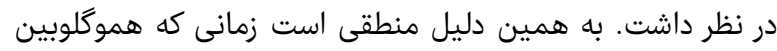

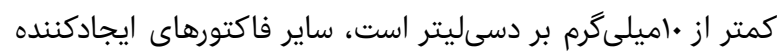

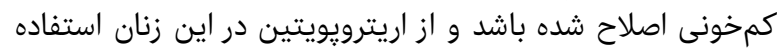

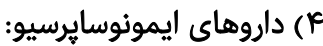

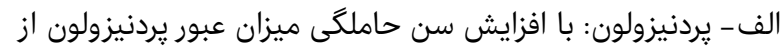

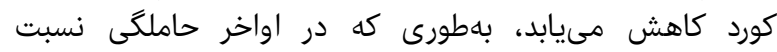

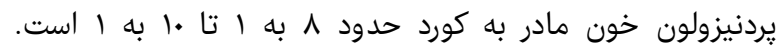

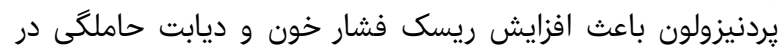

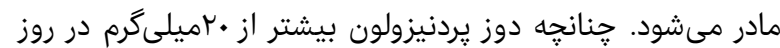

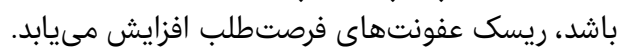

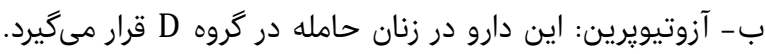

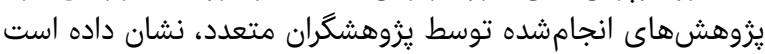

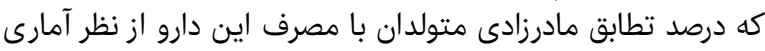

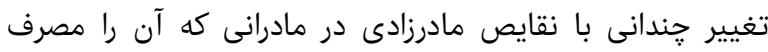

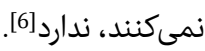

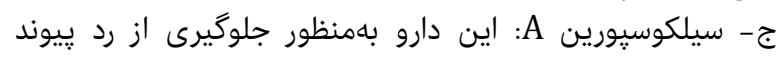

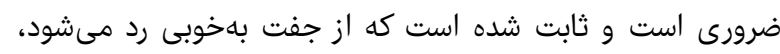

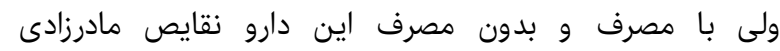

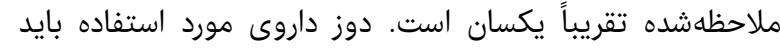

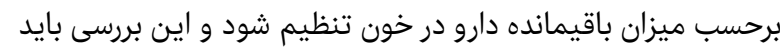

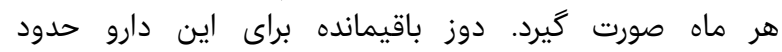

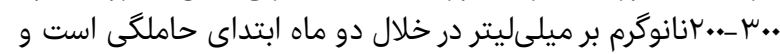

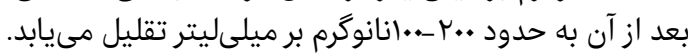

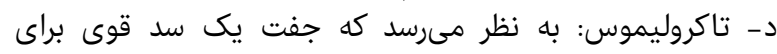

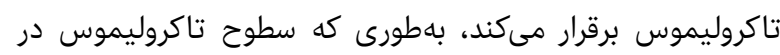

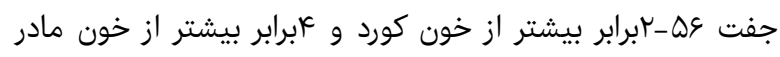

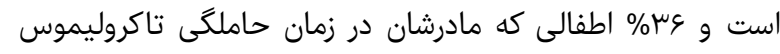

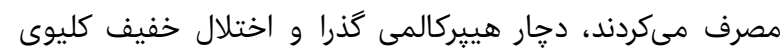

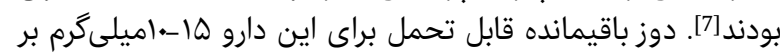

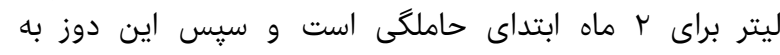

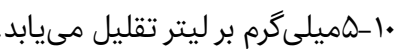

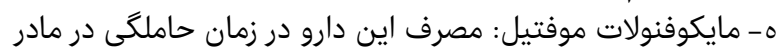

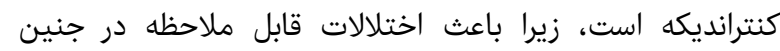

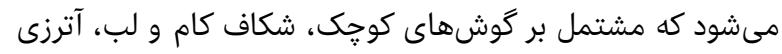

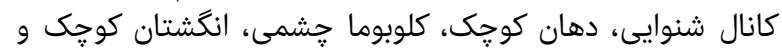

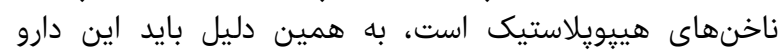

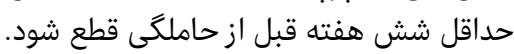

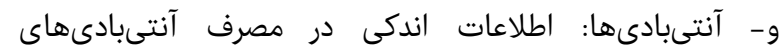

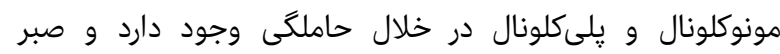

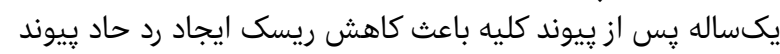

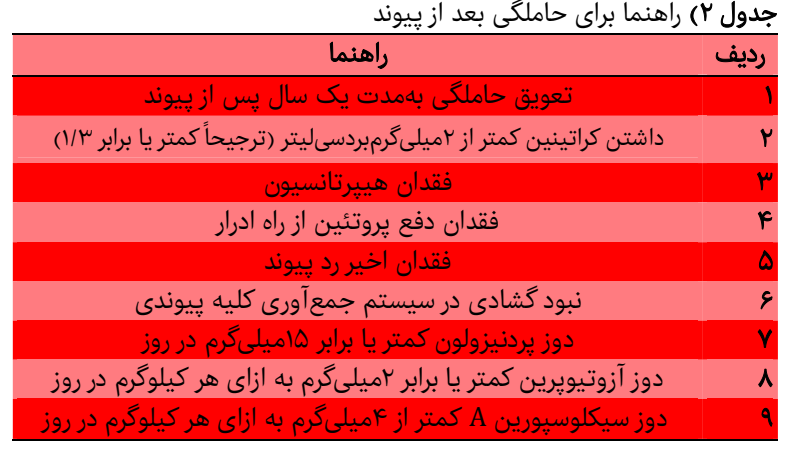

درمان و ملاحظات زنان حامله تحت بي كارند

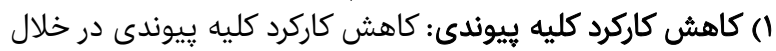

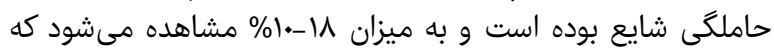

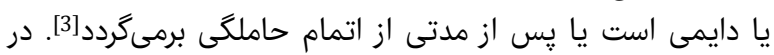

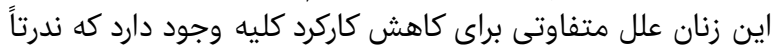

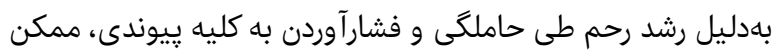

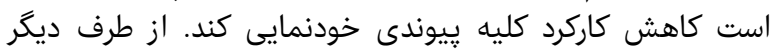

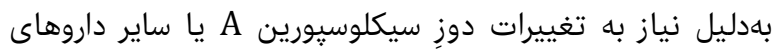

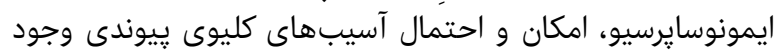

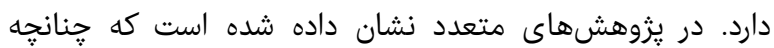

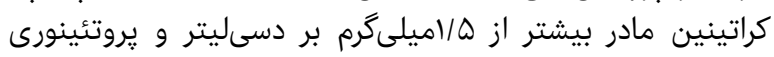

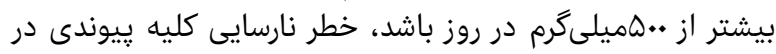

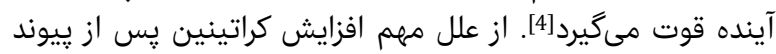

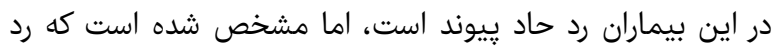

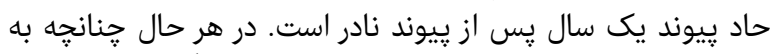

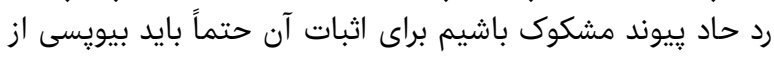

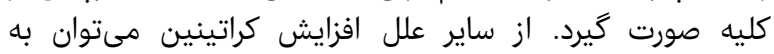

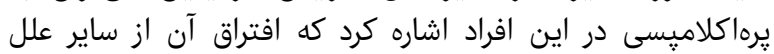

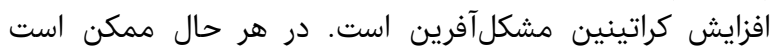

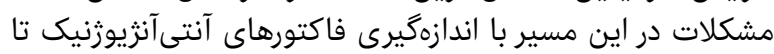

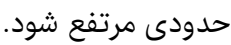

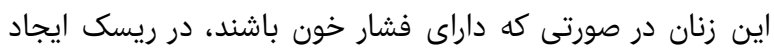

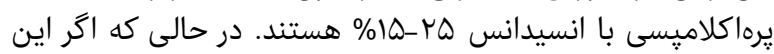

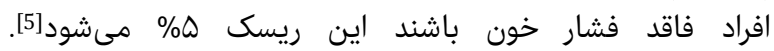

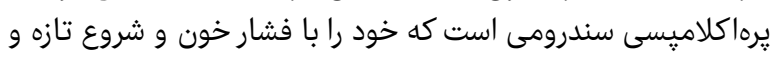

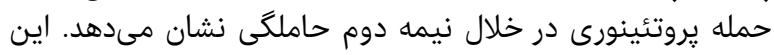

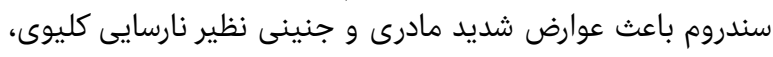

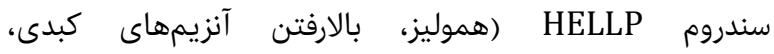

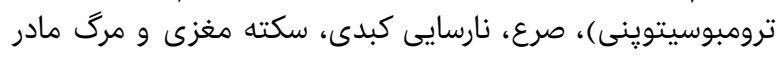

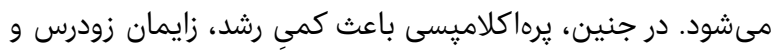

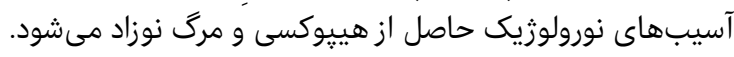

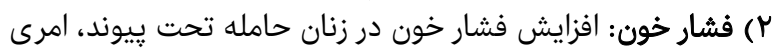

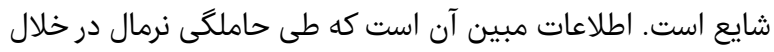

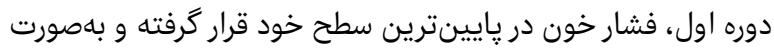

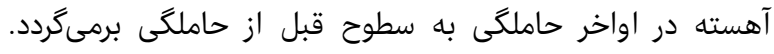

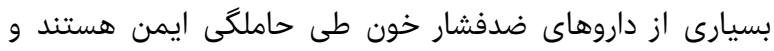

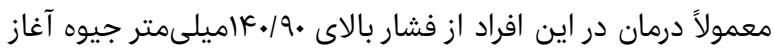

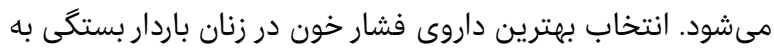

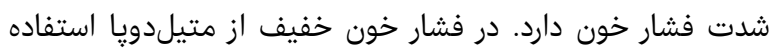

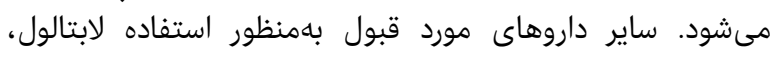
نيفدييين و تيازيدها هستند، ولى نبايد از قاز 
در كودكان مشاهده نشده است، ولى احتياط بر آن است كه از شيردهى تا تكميل اطلاعات امتناع شود.

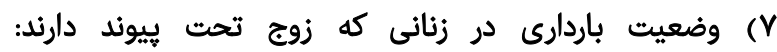

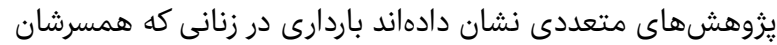

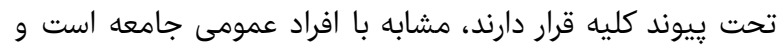

تغيير محسوسى ندارد[10].

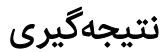

هنوز هم ابهامات زيادى در زمينه يِيوند كليه و عوارض آن در دور دوران

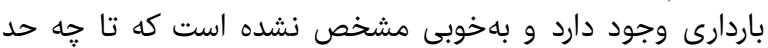

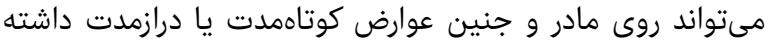

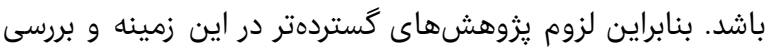
همه جانبه اثرات آن ضرورى به نظر مىرسد.

$$
\begin{aligned}
& \text { تشكر و قدردانى: موردى از سوى نويسندگان ذكر نشده است. }
\end{aligned}
$$

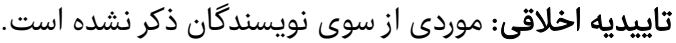

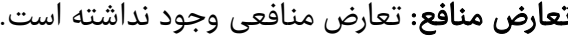

$$
\begin{aligned}
& \text { منابع مالى: موردى از سوى نويسندكان ذكر نشده است. }
\end{aligned}
$$

منابع

1- Mckay DB, Josephson MA. Pregnancy after kidney transplantation. Clin J Am Soc Nephrol. 2008;3(Suppl):S117-25.

2- Armenti VT, Radomski JS, Moritz MJ, Gaughan WJ, Hecker WP, Lavelanet A, et al. Report from the national transplantation pregnancy registry: Outcome of pregnancy after transplantation. Clin Transpl. 2004:10314.

3- Hou s. Pregnancy in renal transplant recipients. Adv Chronic Kidney Dis. 2013;20(3):253-9.

4- Hold PM, Wong CF, Dhanda RK, Walkinshaw SA, Bakran A. Successful renal transplant during pregnancy. Am J Transplant. 2005;5(9):2315-7.

5- Hou S. Pregnancy in renal transplant. Adv Ren Ther. 2003;10(1):40-7.

6- Deshpande NA, James NT, Kucirka LM, Boyarsky BJ, Garonzik-Wang JM, Montgomery RA, et al. Pregnancy outcomes in kidney transplant recipients: A systematic review and meta-analysis. Am J Transplant. 2011;11(11):2388-404.

7- Kukla A, Issa N, Ibrahim HN. Pregnancy in renal transplantation: Recipient and donor aspects in Arab world. Arab J Urol. 2012;10(2):175-181.

8- Amine BH, Haythem S, Kais H, Radhouane R. Pregnancy after renal transplantation: A retrospective study at the military hospital of Tunis from 1992 to 2011. Pan Afr Med J. 2017;28:137.

9- Chakravarty EF, Murray ER, Kelman A, Farmer P. Pregnancy outcomes after maternal exposure to rituximab. Blood 2011;117(5):1499-506.

10- Hutto C, Arvin A. Jacobs R, Steele R, Stagno S, Lyrene R. Intrauterine herpes simplex virus infections. J Pediatr. 1987;110(1):97-101.

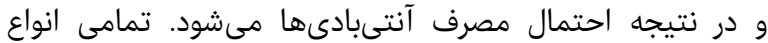

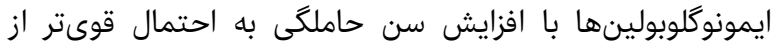

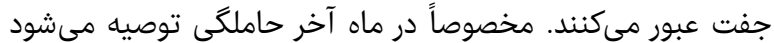

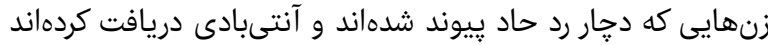

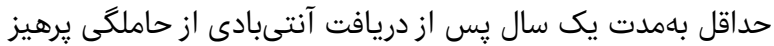

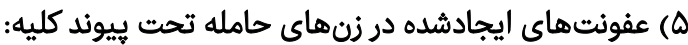

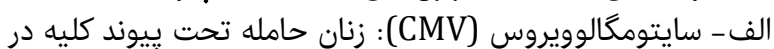

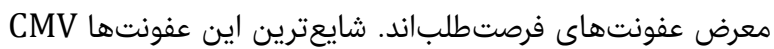

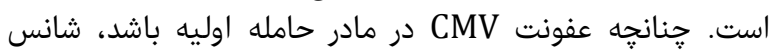

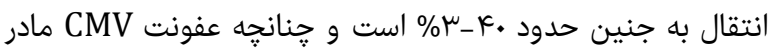

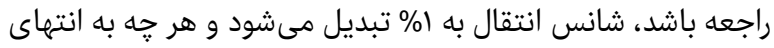

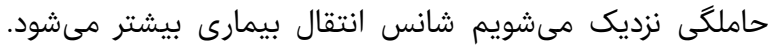

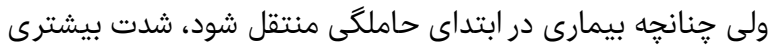

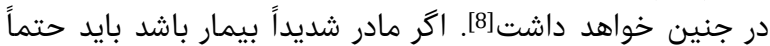

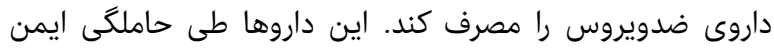

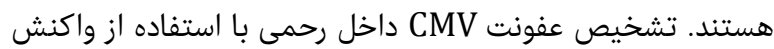

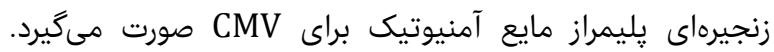

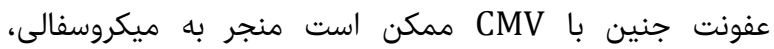

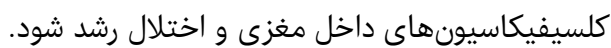

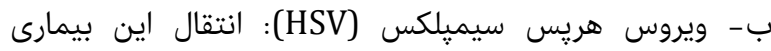

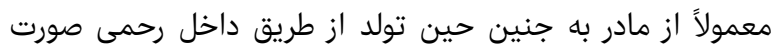

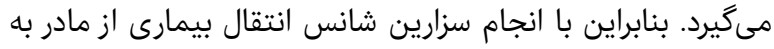

$$
\text { جنين كاهش مى جيابد. }
$$

ج- توكسويلاسموز: عفونت اوليه توكسويلاسمايى مادر حامله، هQ هـ

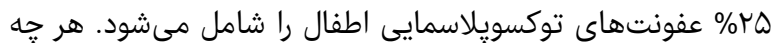

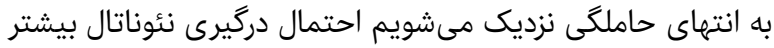

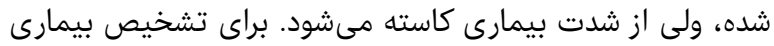

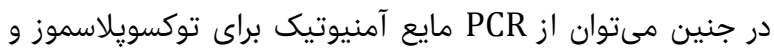

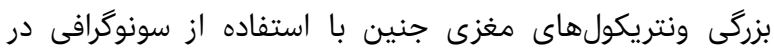

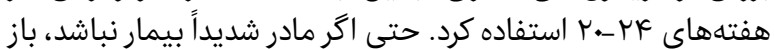

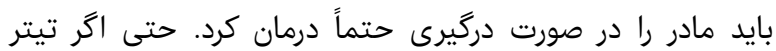

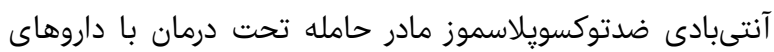

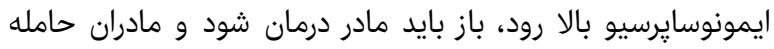

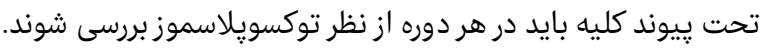

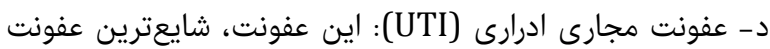

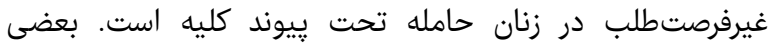

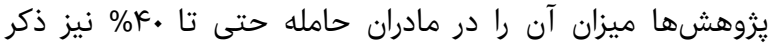

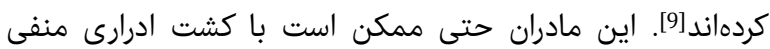

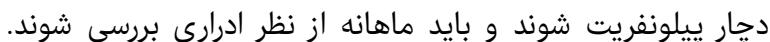

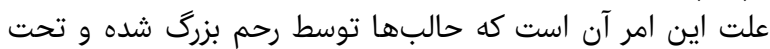

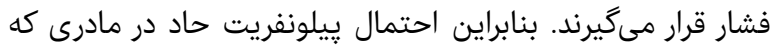

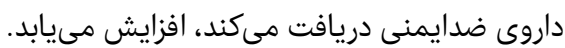

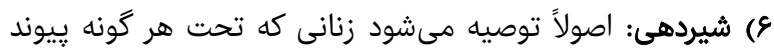

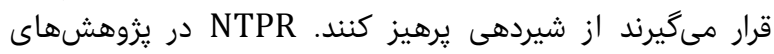

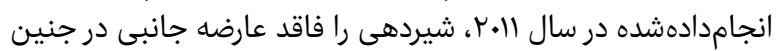

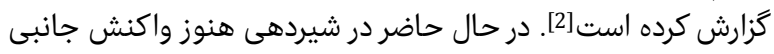

